\title{
Die Ambivalenz der „Abtreibung“
}

\section{Eine Analyse der Kontroverse um den „Schwangerschaftsabbruch“ anhand des Konzeptes zu abweichendem Verhalten von Howard S. Becker}

\author{
Julia BANNWART ${ }^{1}$
}

Universität Fribourg

\section{Einleitung und Fragestellung}

Die Möglichkeit zu einem legalen Abbruch einer ungewollten Schwangerschaft bzw. zu einer Abtreibung ${ }^{2}$ wird in gesellschaftlichen, wissenschaftlichen und politischen Debatten in Anbetracht divergierender Verständnisse über Selbstbestimmung, Menschenrechte, Geschlechterbilder sowie Familie, Beziehung und Sexualität kontrovers diskutiert. Die Praktik der Abtreibung steht dabei im Spannungsfeld moralisierender, feministischer und emanzipatorischer Wertvorstellungen. Hierbei wird das Recht auf den Abbruch einer ungewollten Schwangerschaft als Bestandteil reproduktiver Rechte bzw. von Selbstbestimmungsrechten der Frau, den Rechten des vorgeburtlichen Lebens gegenübergestellt (vgl. Boltanski 2007; Busch 2015). Während der Abbruch einer ungewollten Schwangerschaft lange Zeit illegal und kriminalisiert war, sind die gesetzlichen Rahmenbedingungen für eine Abtreibung heutzutage in der Schweiz besser als je zuvor (Busch 2015:31). Dennoch bleibt dieses Thema mit einem starken Stigma behaftet. In den meisten westlichen Ländern erfolgte die Liberalisierung und die damit einhergehende Legalisierung der Abtreibung in den 1960er und 1970er Jahren, ihre Umsetzung wurde aber unterschiedlich geregelt (Boltanski 2007:14). In der Schweiz ist die Fristenregelung $^{3}$ - sie ermöglicht einen Schwangerschaftsabbruch innerhalb der ersten 12 Wochen

\footnotetext{
${ }^{1}$ Julia Bannwart studiert Soziologie, Sozialarbeit und Sozialpolitik (BA) an der Universität Fribourg und arbeitet als Institutsassistentin an der Hochschule für Soziale Arbeit FHNW. Der vorliegende Artikel baut auf einer Proseminararbeit auf, welche im Rahmen des Proseminars „Devianz, soziale Kontrolle und Strafeinstellungen“ im FS2018 bei Silvia Staubli geschrieben wurde. Kontakt: julia.bannwart@unifr.ch

${ }^{2}$ Im vorliegenden Artikel wird sowohl der Begriff „Abtreibung“ als auch „Schwangerschaftsabbruch“ wertneutral verwendet. Beide Begriffe sind hinsichtlich politischer und moralischer Auseinandersetzungen stark bedeutungsgeladen (Busch 2015:8). Während Abtreibungsbefürworter_innen Begriffe wie „Schwangerschaftsabbruch“ oder „Schwangerschaftsunterbrechung" priorisieren, würden diese aus Sicht von Abtreibungsgegner_innen das "Kind im Mutterleib" und dessen Lebensrecht schon verbal ausmerzen (Kuttner 2008:316). Schmitter (2014:55) zufolge basiert die Unterscheidung dieser beiden Begriffe kulturhistorisch auf unterschiedlichen Deutungen: Der „Schwangerschaftsabbruch“ stellt dabei die legale, die „Abtreibung“ die illegale Form dieser Praktik dar.

${ }^{3} \mathrm{Ab}$ der 13. Woche ist der Schwangerschaftsabbruch gemäss Gesetz nur ausnahmsweise (bei schwerwiegender körperlicher Schädigung bzw. schwerer seelischer Notlage) möglich (Artikel 119 des StGB 1937). In der Schweiz werden die Kosten einer Abtreibung durch die Krankenkassen bezahlt (APAC-Suisse 2012). Von 1942-2002 war ein Abbruch einer ungewollten Schwangerschaft in der Schweiz nur bei Lebensgefahr bzw. bei einem erwarteten schweren Schaden an der Gesundheit der Schwangeren möglich (Schmitter 2014:53-54).
} 
einer Schwangerschaft - seit 2002 im Schweizerischen Strafgesetzbuch verankert (Artikel 119 des StGB 1937). Dies in Anbetracht der Tatsache, dass die Regelung des Schwangerschaftsabbruchs in der Schweiz vorher eines der restriktivsten in Europa gewesen ist (Schmitter 2014:14). Trotz der Legalisierung und Entkriminalisierung der Abtreibung ist seit den 1970er und 1980er Jahren wieder eine zunehmende Moralisierung und Retraditionalisierung in Gang, wodurch diese Thematik ihre Ambivalenz beibehält (Busch 2015:13).

Der vorliegende Artikel geht der Frage nach, wie die theoretische Ausarbeitung von Becker (2014) zu abweichendem Verhalten auf die gesellschaftliche Kontroverse um den Schwangerschaftsabbruch bzw. die Abtreibung angewendet werden kann, und ob sich die „Etikettierungstheorie“4 (vgl. Rössel et al. 2014) auf diesen Tatbestand sinnvoll übertragen lässt. Denn für Becker ist „Abweichung“ nicht etwas, das einer Handlung als solche inhärent ist, sondern wird erst in gesellschaftlichen Zuschreibungsprozessen und interaktiven Aushandlungen hergestellt. Ein solches Muster interaktiver Auseinandersetzung zeigt sich auch in der Kontroverse um den Abbruch einer ungewollten Schwangerschaft: Sie wird von verschiedenen gesellschaftlichen Gruppen mit unterschiedlichen Werten in Bezug gesetzt, wodurch konfligierende Definitionen über diese Praktik entstehen. Entsprechend des interaktionistischen Zugangs Beckers (2014:193-194) in Bezug auf die „Verhaltensabweichung“, sollen hier anhand einer allgemeinen thematischen Darstellung der Abtreibungskontroverse drei Aspekte untersucht werden: Erstens Prozesse, in denen die gegenseitigen Zuschreibungen erfolgen, zweitens Kontexte, in denen sich diese abspielen und drittens die Rolle der an der Interaktion beteiligten Akteur_innen und ihre jeweilige Legitimation sowie Definitionsmacht über „Abweichung“. Es handelt sich hierbei nicht um eine umfangreiche Aufarbeitung dieser Thematik, sondern es werden lediglich spezifische Aspekte von Beckers Ansatz auf ausgewählte Beispiele übertragen. Dabei sollen die gegensätzlichen Positionen in Bezug auf Abweichung nicht als „etwas Besonderes (...), als verdorben oder in irgendeiner magischen Weise besser als andere Verhaltensweisen“, angesehen werden, sondern als ein Verhalten, „das einige missbilligen und andere schätzen“ (Becker 2014:167).

Im folgenden Kapitel werden elementare Aspekte der interaktionistischen Devianzsoziologie von Becker (2014) vorgestellt und auf die Abtreibungskontroverse angewendet. Abschliessend erfolgt ein Fazit, indem die Möglichkeiten und Grenzen in der Anwendung des Ansatzes auf dieses Beispiel diskutiert werden.

\section{Abtreibung als „abweichendes Verhalten“}

In einem ersten Schritt werden zentrale Elemente des Konzeptes von Howard S. Becker zu abweichendem Verhalten dargelegt und der Rahmen für die Analyse der Kontroverse um den Schwangerschaftsabbruch anhand dieses Ansatzes festgelegt. Anschliessend wird die Ambivalenz rund um das Thema Abtreibung aufgezeigt, indem auf den interaktiven Prozess, der sich zwischen den Beteiligten abspielt, eingegangen wird.

\footnotetext{
${ }^{4}$ Becker lehnt die Bezeichnung „Etikettierungstheorie“ ab, da es die ursprünglichen Äusserungen von ihm und anderen nicht verdienen würden, Theorie genannt zu werden (Becker 2014:170).
} 


\section{Abweichendes Verhalten nach Howard S. Becker}

Mit seinem Werk „Aussenseiter" ${ }^{\text {“5 }}$ leistete Howard S. Becker einen entscheidenden Beitrag zur interaktionistischen Devianzsoziologie ${ }^{6}$ und begründete damit die „Etikettierungstheorie“ wesentlich mit (Rössel et al. 2014:7-11). Während Abweichung in anderen devianzsoziologischen Theorien bisher überwiegend als ein Verhalten verstanden wurde, das seinem Wesen nach pathologisch, dysfunktional oder gar die Folge einer psychischen Erkrankung ist, nimmt Becker eine ganz andere Betrachtungsweise auf den Gegenstand abweichenden Verhaltens ein. Becker erfasst Abweichung nicht als ein „fixes“ Objekt respektive als etwas, das einer Handlung inhärent ist, sondern versteht sie als eine „soziale Bedeutung“, die in interaktiven Prozessen hergestellt und zugeschrieben wird (Rössel et al. 2014:17). Demnach ist Abweichung keine dem Verhalten zugrundeliegende Qualität - eine Handlung ist nicht an sich „richtig“ oder „falsch“ bzw. „normal“ oder „unnormal“ -, sondern sie wird erst in konkreten interaktiven Prozessen zwischen Menschen als solches ausgehandelt und definiert, und zwar zwischen dem Menschen, der eine Handlung begeht und denjenigen, die in spezifischer Weise darauf reagieren (Becker 2014:36). Eine abweichende Handlung besteht Becker (2014:171) zufolge aus zwei grundlegenden Elementen: Erstens entsteht sie durch das Begehen einer Handlung, die bestimmte Regeln einer gesellschaftlichen Gruppe bricht bzw. verletzt. Und zweitens muss eine Handlung von dieser Gruppe als abweichend definiert werden. Das abweichende Verhalten ist somit eine Konsequenz aus der Aufstellung und der Durchsetzung von Regeln durch eine gesellschaftliche Gruppe, die bei der Verletzung dieser Regeln die Regelbrechenden sanktioniert und schliesslich als „Aussenseiter“ etikettiert (Becker 2014:31). Etikettieren meint in diesem Zusammenhang den Prozess, in dem eine Handlung durch beteiligte Akteur_innen von aussen als abweichend gekennzeichnet wird (Becker 2014:171). Abweichendes Verhalten ist folglich ein Verhalten, das durch Menschen als solches bezeichnet wird (Rössel et al. 2014:11,31). Da Abweichung erst durch die interaktive Aushandlung als solche definiert wird, können „Abweichende“ nicht als homogene Gruppe erfasst werden, die über gemeinsame, die Abweichung erklärende Merkmale verfügen (Becker 2014:31-32). Der interaktionistische Zugang, den Becker auf Verhaltensabweichung einnimmt, richtet sich einerseits auf die Prozesse, in denen Handlungen als abweichend etikettiert werden, auf die Kontexte, in denen sich solche Zuschreibungen abspielen und letztlich untersucht er die Rolle der an der Interaktion beteiligten Akteur_innen, Ankläger_innen wie Angeklagte (Becker 2014:193-194). Becker nennt diese „moralische Schauspieler“ (Becker 2014:193), und betrachtet ihre jeweilige Legitimation sowie ihre Definitionsmacht über Abweichung

In Anbetracht dessen, dass moderne Gesellschaften über eine grosse Differenzierung hinsichtlich sozialer, ethnischer und kultureller Unterschiede verfügen, bestehen diverse soziale Gruppen, die Verhaltensregeln aufstellen und durchzusetzen versuchen. Diese Regeln geben vor, welche Verhaltensweisen respektive Handlungen in spezifischen Situationen angemessen sind, - welche „richtig“ und welche „falsch“ bzw. akzeptiert und nicht akzeptiert sind - und

\footnotetext{
${ }^{5}$ Das englische Originalwerk "Outsiders. Studies in the sociology of deviance" erschien erstmals 1963.

${ }^{6}$ Howard S. Becker war ein Schüler von Herbert Blumer, der als Begründer des „symbolischen Interaktionismus“ gilt. Becker versteht sein Werk „Aussenseiter“ als eine Fortführung dieser Theorie, indem er sie in die Deviansoziologie einführte (Rössel et al. 2014:8).
} 
welche Sanktionen auf einen Regelverstoss erfolgen können (Becker 2014:25). Die aus vielfältigen Interessen und Wertorientierungen abgeleiteten gesellschaftlichen Regeln können sich gegenseitig widersprechen oder ausschliessen, respektive miteinander in Konflikt geraten (Becker 2014:131). Da Moral- und Wertvorstellungen sowie daraus abgeleitete Normen und Regeln sowohl zeit- als auch kontextabhängig sind, bestehen diverse und sich im Laufe der Zeit ändernde Definitionen darüber, was als abweichend oder als nicht abweichend begriffen wird (Rössel et al. 2014:17-18). Beckers Ansatz zu abweichendem Verhalten konzentriert sich dabei in erster Linie auf die Gruppenregeln, die gegenwärtig wirksam sind, indem sie durch Durchsetzungsversuche aufrechterhalten werden (Becker 2014:26). Regeln - sowohl informelle als auch formelle - die nicht (durch Sanktionen) durchgesetzt werden, verlieren demnach ihre Bedeutung. Ihre Durchsetzung ist selektiv, d.h. Regeln werden in verschiedenen Situationen, bei verschiedenen Personen, zu verschiedenen Zeiten unterschiedlich angewendet (Becker 2014:132). Nach Becker (2014:123) beruht die Regeldurchsetzung zudem auf mehreren Prämissen: Erstens bedarf es einer Initiative bzw. dem Vorhaben in einem „unternehmerischen Akt“ diejenigen zu bestrafen, die eine Regel nicht eingehalten haben. Diese Nichteinhaltung muss des Weiteren für die Regeldurchsetzung in die öffentliche Aufmerksamkeit gelangen. Und letztlich muss dafür ein persönliches Interesse an der Durchsetzung bestehen. Die Durchsetzung ist folglich von der relativen Macht der Akteur_innen und ihrem Zugang zur Öffentlichkeit abhängig (Becker 2014:129). Oftmals sind Situationen sehr komplex, da verschiedene Gruppen an der Definition beteiligt sind, die um die Definitionsmacht konkurrieren. Die Aushandlung der verschiedenen Regeln und damit einhergehend die Aushandlung darüber, was als abweichend angesehen wird, ist somit eine politische Frage (Becker 2014:30).

In diesem Artikel wird vor allem auf die elementaren Aspekte von Beckers Ansatz Bezug genommen: Die interaktionistischen Prozesse, in denen Akteur_innen mit Bezugnahme auf spezifische Werte Regeln aufstellen, durchsetzen und sanktionieren und dadurch Abweichung produzieren. Diese werden auf das Beispiel der Kontroverse um den Schwangerschaftsabbruch angewandt. Andere zentrale Aspekte seiner „Theorie“ werden dabei nicht in die Analyse einbezogen, da sie sich für dieses Beispiel nicht als sinnvoll erweisen ${ }^{7}$ (vgl. Kapitel 3). Im Folgenden werden mögliche Parallelen zwischen der interaktionistischen Perspektive auf abweichendes Verhalten und dem Tatbestand der Abtreibung aufgezeigt und diskutiert.

\section{Die Ambivalenz der "Abtreibung"}

In Bezugnahme auf das Konzept abweichenden Verhaltens von Becker betrachten wir die Abtreibung in erster Linie als „neutrale“ Handlung, die an sich nichts inhärent Abweichendes enthält und erst in Bezugnahme auf spezifische Werte und durch Zuschreibungen in interaktiven Prozessen zu einer abweichenden Handlung erklärt wird. Denn der Abbruch einer ungewollten Schwangerschaft ist aus wertneutraler Perspektive lediglich eine Form der Kontrolle über die Fortpflanzung und kann als weitverbreitete und seit jeher praktizierte menschliche

\footnotetext{
${ }^{7}$ Dies betrifft in erster Linie die Weiterführung des Abweichungskonzeptes von Becker hinsichtlich einer Entwicklung einer „abweichenden Identität“ respektive einer „Karriere abweichenden Verhaltens“, wie er sie anhand einer empirischen Untersuchung von Marihuana-Konsumenten und von Livemusikern herausarbeitet (Becker 2014:57-121). Auch die Verbindung von abweichendem Verhalten und mangelnder Integration in gesellschaftliche Konventionen und Normen sowie die Typen abweichenden Verhaltens können hier nicht berücksichtigt werden (Becker 2014:39-45).
} 
Handlung angesehen werden. Busch (2015:13) zufolge ziehen sich sowohl individuelle als auch gesellschaftliche Versuche, das individuelle Fortpflanzungs- und Sexualverhalten zu regulieren und zu beeinflussen, durch die Geschichte der Menschheit. Boltanski (2007) schreibt dem gezielten Abbruch einer ungewollten Schwangerschaft einen universalen Charakter zu, da er sowohl in traditionellen als auch in modernen Gesellschaften nachweislich praktiziert worden ist bzw. praktiziert wird. Die Möglichkeit zur Abtreibung sei dem menschlichen Dasein in einer Gesellschaft daher grundlegend (Boltanski 2007:35). Boltanski arbeitet jedoch spezifische Eigenschaften über die Praktik der Abtreibung heraus, die wesentlich auf bestehende Ambivalenzen dieser Thematik hinweisen und denen er eine allgemeine Gültigkeit beimisst. Ihm zufolge stellt die Abtreibung trotz ihrer universalen Gültigkeit in zahlreichen Gesellschaften einen „Gegenstand der Missbilligung“ (Boltanski 2007:39) dar. Trotz dieser Missbilligung, und trotz überwiegender formeller oder informeller Sanktionen sei aber wiederum eine grosse Toleranz für den gewollten Schwangerschaftsabbruch vorhanden (Boltanski 2007:39). Des Weiteren finde die Abtreibung - obwohl universal bekannt und häufig praktiziert - normalerweise im Geheimen oder Verborgenen statt, wodurch sie ihre zweideutige Position zwischen dem Erlaubten und Verbotenen beibehalte (Boltanski 2007:40-49). In diesem Zusammenhang zieht Boltanski (2007:40-41) die Unterscheidung des „Offiziellen“ und des „Offiziösen“8 heran, die sich auch im Hinblick auf gesellschaftliche Zuschreibungs- bzw. Etikettierungsprozesse (vgl. Beckers) als sinnvoll erweist. Dabei wird die „offizielle Welt“ - die Welt des schriftlich festgelegten Rechts, der Religion, der Politik, der Öffentlichkeit und der Welt des Äusseren - als Welt der Männer, und die „offiziöse Welt“ - der Bereich des Verborgenen, des Privaten und des Inneren, der Bereich der Schwangerschaft und Geburt, des Hauses - als die Welt der Frauen unterschieden. Während der Bereich des „Offiziellen“ einen öffentlichen und kollektiven Charakter habe, sei der Bereich des „Offiziösen“ mit etwas Schändlichem und Illegalem behaftet. Die Abtreibung ist demnach, solange sie im Bereich des „Offiziösen" stattfindet, stillschweigend akzeptiert und wird erst dann zum Gegenstand der Empörung, wenn sie in den Bereich des „Offiziellen“ rückt. Entsprechend der Tatsache, dass die Abtreibung - unabhängig von ihrer gesellschaftlichen Akzeptanz respektive ihrer Legalität seit jeher praktiziert wird, aber in der offiziellen gesellschaftlichen Debatte mit solcher Brisanz thematisiert wird, scheint die Heranziehung dieser Unterscheidung als plausibel. Wenn wir diesen Tatbestand mit der „Etikettierungstheorie“ in Bezug setzen, dann könnte dies auf Beckers Annahme hinweisen, dass der vollständige Prozess zur Entstehung abweichenden Verhaltens über das Begehen einer „regelbrechenden“ Handlung hinaus, eine offizielle Zuschreibung erfordert. Solange sie im „offiziösen“ Bereich stattfindet, handelt es sich um eine „geheime Abweichung“ (Becker 2014:39). Erst im Bereich des „Offiziellen“ bzw. in der öffentlichen Aufmerksamkeit und im „unternehmerischen Akt“ (Becker 2014:129) gesellschaftlicher Gruppen, gerät die Praktik der Abtreibung in das Spannungsfeld der „persönlichen“ Interessen und Regeln, die zu ihrer Aufrechterhaltung einer Durchsetzung durch Sanktion bedürfen und schliesslich zu Etikettierungsprozessen führen.

\footnotetext{
${ }^{8}$ Bourdieu hat die Unterscheidung dieser beiden Bereiche in seinen ethnologischen Studien über die Kabylische Gesellschaft respektive in seinem Werk „Entwurf einer Theorie der Praxis auf der ethnologischen Grundlage der kabylischen Gesellschaft“ (1979) eingehend analysiert und mitgeprägt.
} 
Der absichtliche Schwangerschaftsabbruch bzw. die Abtreibung wird von verschiedenen gesellschaftlichen Gruppen unterschiedlich interpretiert und mit unterschiedlichen Werten in Bezug gesetzt. Bezugnehmend auf Beckers Konzept handelt es ich dabei um einen Etikettierungsprozess, bei dem in einer interaktiven Auseinandersetzung zwischen beteiligten Gruppen um die Definitionsmacht darüber gerungen wird, welche Regeln durchgesetzt, was als abweichend definiert wird und welche Sanktionen auf einen Regelbruch folgen. Dass Moralund Wertvorstellungen sowie daraus abgeleitete Normen und Regeln sowohl zeit- als auch kontextabhängig sind - und somit auch die Definitionen darüber, was als abweichend oder nicht abweichend begriffen wird, veränderbar sind -, zeigt sich bei der Abtreibungskontroverse beispielhaft (Rössel et al. 2014:17-18). Denn die Vorstellungen und Bedeutungen von Schwangerschaft und Schwangerschaftsabbruch unterliegen sowohl gesellschaftlich als auch individuell historischen Veränderungen (Hahn 2015:41). Da es im Rahmen dieses Artikels nicht möglich ist, einen umfangreichen Überblick über die historischen Entwicklungen hinsichtlich der sich wandelnden Werteorientierungen und daraus abgeleiteten Regelungen und Gesetzgebungen aufzuzeigen, beschränkt sich die folgende Darlegung auf einzelne Aspekte dieser Thematik. Es geht also darum, verschiedene Akteur_innen und ihre Bezugnahme auf unterschiedliche Werte im Zusammenhang mit dem Thema Abtreibung an einzelnen Beispielen aufzuzeigen. Im Folgenden wird die Praktik des Schwangerschaftsabbruchs hauptsächlich aus zwei gegensätzlichen Perspektiven beleuchtet - einerseits aus einer christlichkonservativen und andererseits aus einer feministischen Sichtweise. Da beide Seiten je spezifische ethische Grundsätze verfolgen, können sowohl Abtreibungsgegner_innen als auch Abtreibungsbefürworter_innen als „Kreuzzüge unternehmende Reformer“ (Becker 2014:146) angesehen werden, die in ihrer Regelaufstellung humanitäre Motive verfolgen. Nach Becker (2014:149-151) entstehen aus solchen „moralischen Kreuzzügen“ - wenn sie erfolgreich sind - neue Regeln und damit einhergehend auch eine „Durchsetzungs-Maschinerie“ (Becker 2014:149) und ihre Institutionalisierung.

Wie noch aufgezeigt wird, bildet die eintretende Liberalisierung der Abtreibungsgesetzgebung ab den 1960er Jahren einen entscheidenden Wendepunkt für die Durchsetzung und Institutionalisierung der Abtreibung. Während vorher die Abtreibung an sich, sowie ihre Befürwortenden der Abweichung bezichtigt und von einer verhältnismässig dominanten Gegenseite zu "Aussenseitern“ gemacht wurden, so sind die Argumente der Abtreibungsgegner_innen heutzutage eher befremdlich, aber dennoch, zumindest in informeller Weise, wirksam. Mit der Entstehung von Regeln und ihrer Durchsetzung für die Abtreibung entstand somit auch eine neue Gruppe von „Aussenseitern“. Dass die inzwischen selbst zu „Aussenseiter“ gewordenen Abtreibungsgegner_innen nach wie vor versuchen, „eine Doktrin zu verfechten und zu predigen, die im Laufe der Zeit immer seltsamer klingt“ (Becker 2014:151) wird im Folgenden noch aufgezeigt. Auf die Rolle der Frau, die eine Abtreibung durchführt und dadurch je nach Perspektive den Status einer „Aussenseiterin“ annimmt, wird im zweiten Teil des Kapitels eingegangen. 


\section{Das Konkurrieren um die Deutungsmacht über den Schwangerschaftsabbruch}

Bis Mitte des 20. Jahrhunderts war der Abbruch einer ungewollten Schwangerschaft in den westlichen Ländern ${ }^{9}$ kriminalisiert und unterlag beträchtlichen Sanktionen ${ }^{10}$. Neben den christlichen und konservativen Vorstellungen über Sexualität und Reproduktion waren es vor allem auch bevölkerungspolitische Interessen - Befürchtungen hinsichtlich Demographie und Geburtenraten -, die das Abtreibungsverbot legitimierten. Denn die Regulierung und Kontrolle der Bevölkerung sowie deren Wachstum bildeten ein wichtiges Feld der staatlichen Politik (Hahn 2015:45). Ab den 1960er Jahren verloren diese bevölkerungspolitischen Legitimationsmuster an Bedeutung und verschoben ihren Fokus auf die individuelle Ebene, insbesondere auf die psychischen Folgen und die negativen Auswirkungen einer Abtreibung (Hahn 2015:48). Die Frau wurde als hilfsbedürftige Person dargestellt, die in ihrer Entscheidungsfindung Unterstützung benötigt (Hahn 2015:49). Dadurch wurden Verhaltensnormen konstruiert, nach denen die negativen psychischen Folgen „gesunden“ und „normalen“ Frauen zugeordnet wurden, während Frauen ohne Verarbeitungsprobleme nach einer Abtreibung als unweiblich und unsensibel galten (Hahn 2015:49). Das Abtreibungsverbot bzw. die restriktive Handhabung bei einer Abtreibung wurde mit dem Schutz der Frau vor Folgeschäden legitimiert. Eben diese „patriarchalen Strukturen der Gesellschaft und die stereotypen Vorstellungen von Mütterlichkeit und Weiblichkeit“ (Hahn 2015:55) wurden von den Frauenrechtler_innen bzw. feministischen Bewegungen, als wichtigste Vertreter_innen der Abtreibungsbefürworter_innen, ab den 1960er und 1970er Jahren grundlegend in Frage gestellt. Sie haben sowohl in den USA als auch in Europa bzw. der Schweiz entscheidend zur Entkriminalisierung und Legalisierung der Abtreibung beigetragen. Die zentralen Forderungen dieser Bewegung bildeten den Anspruch auf Autonomie und Selbstbestimmung, körperliche Integrität sowie die Gleichberechtigung der Frau. Aber auch die Liberalisierung der Beziehungsweisen, Freiheit in der Sexualität und damit einhergehend auch die Verhütung und Abtreibung waren Teil dieser Forderungen, mit dem Ziel, die konservativen Strukturen aufzubrechen (Schmitter 2014:3; Busch 2015:23). Sie sahen die Kontrolle über die weibliche Reproduktion und die Möglichkeit zu einem legalen und sicheren Abbruch einer Schwangerschaft als konstitutiv, damit Frauen die Kontrolle über ihr eigenes Leben erlangen konnten, um umfänglich an der Gesellschaft partizipieren zu können (Schmitter 2014:3). Unter den Slogans „ob Kinder oder keine entscheiden wir alleine“ oder „Mein Bauch gehört mir“ (Busch 2015:23) forderten sie die Legalisierung der Abtreibung sowie die entsprechende medizinische Versorgung. Ein zentrales Element der feministischen Bewegung bildete die Kritik an gesellschaftlich produzierten männlichen Privilegien, die Frauen den Männern strukturell unterordnen würden, und damit einhergehend die Veränderung der gesellschaftlichen Machtverhältnisse (Schmitter 2014:23). Dabei verstanden sie ihr Handeln als politisches Handeln, bei dem sie aus dem ihnen zugewiesenen Bereich des "privaten“ und „apolitischen“ in den Bereich des Offiziellen eindringen wollten (Schmitter 2014:24-27). In der Schweiz konnte sich die gesetzliche Legali-

\footnotetext{
${ }^{9}$ Die dargestellten Abläufe und Entwicklungen im Zusammenhang mit der Kontroverse um den Schwangerschaftsabbruch gelten sowohl für die USA als auch für Europa. Dabei wird eine sehr grobe Übersicht dargelegt, die einzelnen Entwicklungen und länderspezifischen Eigenheiten werden im Rahmen dieses Artikels nicht aufgezeigt.

${ }^{10}$ Somit handelte es sich bei der Abtreibung um Delinquenz: Eine spezifische Ausprägung von Devianz, bei welchem das abweichende Verhalten gegen Gesetze verstösst (Lamnek 2013:49).
} 
sierung der Abtreibung erst 2002 mit der Fristenregelung im Schweizerischen Strafgesetzbuch verankern (Artikel 119 des StGB 1937). Seit den 1970er und 1980er Jahren ist in den westlichen Ländern jedoch wieder eine zunehmende Moralisierung und Retraditionalisierung in Gang, die die Ambivalenz dieser Thematik weiterhin erstarken lässt (Busch 2015:13). Die Rolle der Kirche und die Verbindung von Moral, Politik, Recht und Religion sowie extrem konservative Werte hinsichtlich Familie, Sexualität und Beziehung bilden die treibenden Kräfte in der Behinderung der Anerkennung des Rechts auf einen Schwangerschaftsabbruch und damit einhergehend auch des Zugangs $\mathrm{zu}$ entsprechender medizinischer Versorgung (Busch 2015:17-18). Eine zentrale Forderung der Abtreibungsgegner_innen als „Anwälte des ungeborenen Lebens“ (Kuttner 2008:505) bzw. von „Lebensrechtsbewegungen“ (Schmitter 2014:3) ist es, den Schutz des vorgeburtlichen Lebens dem Recht der Frau auf einen Abbruch einer ungewollten Schwangerschaft vorzuziehen (Busch 2015:14). Dabei werden dem embryonalen Leben ab seiner Entstehung Rechte zugesprochen, wodurch der Fötus bzw. Embryo zu einem eigenständigen Wesen mit Persönlichkeitsrechten und zu einem "Grundrechtsträger" (Busch 2015:28) konstruiert wird, der ein Recht auf Schutz und „Menschenwürde“ (Schmitter 2014:3) habe. Aus dieser Sichtweise ist die Abtreibung eines ungeborenen Kindes mit Mord gleichzusetzen und der Abtreibungsvorgang - beispielweise das „Zerquetschen“ und „Verschneiden“ des „Kindes“ „ohne Betäubung“ (Kuttner 2008:314) - wird als ein grausames und unmenschliches Verfahren wahrgenommen. Zu den „Tötungen“ von „Kindern“ durch Schwangerschaftsabbrüche können aus dieser Sicht auch die Fälle einbezogen werden, in denen eine Schwangerschaft durch Medikamente verhindert wird, wie bei Ovulationshemmern (bspw. Antibabypille, „Pille danach“) (Kuttner 2008:314).

Mit welchen dramatischen Anklagen die Praktik der Abtreibung bzw. ihre Befürwortenden konfrontiert sein können, zeigt das folgende Zitat aus dem Text von Kuttner (2008) „Zur Soziologie der Abtreibung“ - aus einer extremen christlich-konservativen Sichtweise - beispielhaft:

Die liberalen Abtreibungsgesetze können nur in einer Gesellschaft eingeführt werden, welche einen Entchristianisierungsprozess durchgemacht hat, der die philosophische Basis der moralischen Werte zerstört hat. Die entchristliche Gesellschaft, ohne Sicherheiten und ohne bleibende Werte, wird eine leichte Beute der irrenden Ideologien. Der Schwachpunkt dieser Ideologien besteht darin, dass sie auf Lügen aufgebaut sind und im Gegensatz zu den wissenschaftlichen Erkenntnissen über das Leben vor der Geburt stehen, (...). Die Geschichte über Rassismus und Marxismus hat gezeigt, dass diese lügnerischen Ideologien höchstens zwei oder drei Generationen dauern, und dann unter dem Gewicht der eigenen Falschheit und den sozialen Katastrophen ihrer Fehler zusammenstürzen. (Kuttner 2008:315-316)

Auch bringt Kuttner in seinem Text bevölkerungspolitische Argumentationen mit ein, indem er sich um die „kinderarme Gesellschaft“ sorgt, die „ihre sozialstaatlichen Mittel und allmählich Millionenbeträge aus der zerrütteten Staatskasse weiterhin dafür verwendet, um Hunderttausende ungeborener Kinder am Weiterleben zu hindern, auf diese Weise kommende Generationen existenziell in ihrer Lebensgrundlage zu schädigen und sich selbst für eine ausreichende Altersversorgung ausserstand zu setzen“ (Kuttner 2008:318). Als Massnahmen plädiert Kuttner (2008) für eine Wiedereinführung der Tugend und Keuschheit und die morali- 
sche Disziplinierung der Jugendlichen, um ihre „sexuellen Triebkräfte“ (Kuttner 2008:323) zu regulieren.

\section{Die „abtreibende“ Frau als „Aussenseiterin“?}

Becker zufolge wird ein Mensch durch das Nicht-Befolgen von Regeln, auf welche sich eine Gruppe geeinigt hat und anwendet, zum „Aussenseiter“ (Becker 2014:25). Eine Frau, welche eine Abtreibung vornimmt, ist demzufolge je nach Perspektive mit der Zuschreibung der Abweichung konfrontiert und kann somit zur „Aussenseiterin“ werden. Zwar kann heutzutage auf legalem Wege eine Abtreibung durchgeführt werden, wodurch keine gesetzlichen Sanktionen befürchtet werden müssen. Menschen bzw. in diesem Fall Frauen sind jedoch vielen verschiedenen Gruppen zugehörig, die über je eigene Regeln verfügen, und so können auch informelle Regeln und Sanktionen wirksam sein (Becker 2014:30). Da die Abweichung erst im interaktiven Prozess erfolgt, bilden nach Becker die in diesem Prozess als abweichend „Etikettierten" keine homogene Gruppe, die über bestimmte gemeinsame Merkmale hinsichtlich ihrer Persönlichkeit oder ihrer Lebenssituation verfügen, welche die Abweichung erklären könnten (Becker 2014:31-32). Ausser dem Tatbestand einer bestimmten durchgeführten Handlung - in diesem Fall der Schwangerschaftsabbruch - werden Menschen erst durch den Zuschreibungsprozess durch eine gesellschaftliche Gruppe als Abweichende definiert. Dies lässt sich gut auf das Beispiel der Abtreibung übertragen, da diese heutzutage von Frauen aus unterschiedlichen Bildungs- und sozialen Schichten durchgeführt werden ${ }^{11}$.

Grundsätzlich ist es sehr wahrscheinlich, dass eine Frau bei ihrer Entscheidung für oder gegen eine Schwangerschaft mit grossen emotionalen und ethischen Konflikten konfrontiert ist, da die Praktik der Abtreibung sehr zentrale Fragen nach der menschlichen Existenz und der Verfügungsmöglichkeit darüber berührt (Busch 2015:34). Für Abtreibungsbefürworter_innen ist das Recht auf „reproduktive und sexuelle Selbstbestimmung“ (Busch 2015:35) erst dadurch umgesetzt, wenn eine Frau eine Abtreibung vollziehen kann, ohne sich dafür schuldig zu fühlen bzw. schuldig gesprochen zu werden. Eine gute Entscheidung kann demnach nur in einem Rahmen erfolgen, in dem die Gesellschaft die Abtreibung anerkennt, ohne Sanktionen und moralisierende Diskurse. Wenn die Abtreibung einem gesellschaftlichen Stigma unterliegt, sei die Frau gezwungen, sich moralisch zu rechtfertigen, was ein Schulderleben und eine konflikthafte Verarbeitung der Abtreibung fördere (Busch 2015:36). Die Gegenseite argumentiert zwar auch mit schützenden Absichten für die Frau, die ungewollt schwanger ist. Dabei soll jedoch die Frau vor der Abtreibung geschützt werden, da sie durch mögliche Spätfolgen ${ }^{12}$ stark geschädigt werden könne (Kuttner 2008:303). Der Schutz der Frau müsse des Weiteren dahingehend erfolgen, dass sie durch das Verbot der Abtreibung vor einer Entscheidung mit existenzieller Tragweite geschützt werde (Kuttner 2008:318). Das Recht

\footnotetext{
${ }^{11}$ Busch (2015:37) zufolge spielen heute vor allem die sich wandelnden Lebensbedingungen hinsichtlich des Rollenverständnisses der Frau, ihren beruflichen Herausforderungen sowie den Lebensansprüchen eine entscheidende Rolle in der Entscheidung für oder gegen eine ungeplante Schwangerschaft.

${ }^{12}$ Schweiger (2015:247) zufolge werden psychische Aspekte eines Schwangerschaftsabbruchs von den Abtreibungsgegner_innen immer wieder instrumentalisiert, um ihre Gegenseite zu legitimieren. Bspw. wurde das „Post Abortion Syndrom“ - angeblich eine spezifische Form der posttraumatischen Belastungsstörung - als mögliche Folge eines Schwangerschaftsabbruchs konzeptualisiert. Obwohl dieses Syndrom weder psychologisch noch medizinisch anerkannt ist, hält sich dieses Thema hartnäckig in der Debatte um den Schwangerschaftsabbruch.
} 
der Frau auf eine eigenständige Entscheidung wird ihr von dieser Seite vollständig abgesprochen.

Letztlich ist es individuell, ob eine Abtreibung als eine Chance oder als schwerwiegender Schicksalsschlag empfunden wird (Busch 2015:35). Die bestehenden gesellschaftlichen und politischen Diskurse tragen auf jeden Fall nicht zu einer verbesserten Entscheidungssituation bei, wobei „Akteure häufig selbst uneins darüber sind, was abweichend ist“ (Becker 2014:174).

Beckers Aspekt des „Regelbruchs“ (2014:45) aufgrund einer mangelnden Integration in gesellschaftliche Konventionen und Normen kann für die gegenwärtige Zeit nicht auf das Beispiel der Abtreibung übertragen werden; Abtreibung ist heutzutage von Gesetzes wegen straffrei und nicht auf eine anormale Entwicklung der Betroffenen in der Gesellschaft zurückzuführen. Die einzige Alternative neben der Abtreibung ist die Akzeptanz der Schwangerschaft und die Geburt des Kindes (mit der Möglichkeit der Adoption). Die Abtreibung kann somit nicht primär als einen „inneren Impuls einen ungesetzlichen Weg zur Lösung ihrer Probleme zu suchen" (Becker 2014:45) verstanden werden. Auch macht die Weiterführung des Abweichungskonzeptes von Becker hinsichtlich einer Entwicklung einer "abweichenden Identität“ (Becker 2014:43) respektive einer Karriere abweichenden Verhaltens in diesem Beispiel wenig Sinn, da die Abtreibung oftmals lediglich ein einmaliges Ereignis im Leben einer Frau darstellt, die abgesehen von dieser Handlung "normal“ in die Gesellschaft und ihre Konventionen eingebunden sein kann (Becker 2014:49). Allerdings könnte die Feststellung Beckers, dass das Individuum aus der Unfähigkeit heraus, die Erwartungen anderer zu erfüllen, abweichende Wege einschlägt (vgl. Becker 2014:52), möglicherweise herangezogen werden, um die Entscheidung zu einer Abtreibung zu erklären. Denn gerade hinsichtlich der Mutterrolle bestehen unzählige normative Vorstellungen, die mit sehr grossen gesellschaftlichen Erwartungen verbunden sind, und somit zu grossen Versagensängsten hinsichtlich der werdenden Mutterschaft beitragen können.

\section{Möglichkeiten und Grenzen des Ansatzes am Beispiel der Abtreibung}

Dass abweichendes Verhalten erst als solches definiert wird, wenn ein Regelbruch öffentlich kommuniziert und sanktioniert wird, kann am Beispiel der Abtreibung und ihren unterschiedlichen Wahrnehmungen - je nachdem ob sie im offiziellen oder im offiziösen Kontext stattfindet - sinnvoll begründet werden. Im offiziösen Bereich bildet sie demnach lediglich eine "geheime Abweichung" (Becker 2014:39), die (noch) nicht sanktioniert wird, jedoch dadurch ihre zweideutige Stellung beibehält. Auch die Bezeichnung der „Kreuzzüge unternehmenden Reformer“ (Becker 2014:146), die sich auf humanitäre Motive beziehen, scheint im vorliegenden Beispiel passend. So konnten die Abtreibungsbefürworter_innen die Legalisierung der Abtreibung und deren gesetzliche Regelung bewirken. Grundsätzlich ist es den Abtreibungsbefürworter_innen gelungen, ihre Deutungsmacht durchzusetzen und die Abtreibung zu entkriminalisieren. Bezugnehmend auf Beckers Konzept wurden bei diesem Prozess die entscheidenden Prämissen (vgl. Becker 2014:129) für eine Institutionalisierung von Regeln erfüllt: Aufgrund eines hartnäckigen und langfristigen Engagements wurden die feministischen Forderungen in die Öffentlichkeit getragen, wo sie eine mächtige Gegenkraft zu den bestehenden gesellschaftlichen und gesetzlichen Verhältnissen bilden konnten. Somit 
konnten sie ihre Werte hinsichtlich der Autonomie und (sexueller) Selbstbestimmung gegen die Werte der Gegenseite zumindest formell durchsetzen und gesetzlich verankern. Das Merkmal Geschlecht im Zusammenhang mit Macht respektive gesellschaftlichen Machtverhältnissen spielte in der Durchsetzung feministischer Forderungen eine entscheidende Bedeutung (vgl. Becker 2014:38). Die Forderungen um die Rechte der Frau, und damit einhergehend auch die Rechte auf den Abbruch einer ungewollten Schwangerschaft, wurden somit zu einem "Gegenstand von Konflikt und Auseinandersetzung“ (Becker 2014:38) in politischen Prozessen. Mit der Entstehung von Regeln und ihrer Durchsetzung für die Abtreibung entstand somit auch eine stark vertretene Perspektive, die die Abtreibungsgegner_innen als eine neue Gruppe von „Aussenseitern“ betrachtet. Dass die inzwischen selbst zu „Aussenseitern“ gewordenen Abtreibungsgegner_innen nach wie vor versuchen, „eine Doktrin zu verfechten und zu predigen, die im Laufe der Zeit immer seltsamer klingt“" (Becker 2014:151), zeigt der Text von Kuttner (2008) beispielhaft. Die massiven Zuschreibungsprozesse der Gegenseite, die die Abtreibung mit Tötung gleichsetzen, tragen aber auch heute noch dazu bei, dass der Abbruch einer ungewollten Schwangerschaft mit einem massiven Stigma behaftet ist und seine ambivalente und tabuisierte Position beibehält. Dies kann vor allem auf die Frauen, die eine Abtreibung vornehmen, dramatische Auswirkungen haben, da sie informellen Sanktionen ausgesetzt sein können.

Im vorliegenden Artikel konnte der Ansatz von Becker zu abweichendem Verhalten in einigen Aspekten sehr sinnvoll auf den Tatbestand der Abtreibung angewendet werden. So zeigen sich die Auswirkungen von gesellschaftlichen Aushandlungen und die daraus resultierenden Konsequenzen beispielhaft. Die Etikettierungstheorie liefert keine Erklärung für die Ursachen von Verhaltensabweichungen, denn die Frage nach den Ursachen würde einheitliche „Abweichungskategorien“ (Rössel et al. 2014:19) verursachen und dies ist aufgrund der Annahme, dass die Definitionen erst im Konflikt entstehen und sowohl pluralisiert als auch prozessualisiert sind, nicht möglich.

Auf die Typen abweichenden Verhaltens von Becker (2014:39) wurde hier nicht weiter eingegangen, da sie sich im Zusammenhang mit der gewählten Thematik nur bedingt sinnvoll einsetzen liessen ${ }^{13}$. Des Weiteren wurden, wie bereits erwähnt, die Aspekte für eine abweichende Karriere respektive Identität nicht weiterverfolgt. Abtreibung ist eine Handlung, die zwar mit individuellen „Gegebenheiten der Sozialstruktur wie auch Änderungen der Einstellungen, Motivationen und Wünsche“ (Becker 2014:43) in Zusammenhang steht, aber von Frauen aus allen Schichten, aufgrund sehr unterschiedlicher Gründe durchgeführt wird und oftmals ein einmaliges Erlebnis darstellt.

Die häufig geäusserte Kritik an Beckers Ansatz, dass er sich in einigen Aspekten selbst widersprechen würde, muss zwar berücksichtig werden, ist aber insofern unwirksam, indem er selbst keinen Anspruch auf eine Theorieentwicklung erhebt und „lediglich“ einen Blickwinkel

\footnotetext{
${ }^{13}$ Wenn die Abtreibung im Bereich des Offiziösen stattfindet, so wurde hier auf die „geheime Abweichung“ verwiesen. Dieser Abweichungstyp, wie ihn Becker konstruiert hat, wurde von mehreren Kritiker_innen in Frage gestellt, da die Abweichung Becker zufolge erst mit der „offiziellen“ Aushandlung als solche definiert wird und die Handlungen unabhängig von den "gefällten Urteilen“ (Becker 2014:176) bestehen. Becker reagierte auf diese Kritik mit der Begründung, dass die geheime Verhaltensabweichung darin bestehe, „empfindlich zu sein für die gewöhnlich benutzten Verfahren zur Aufdeckung von Verhaltensabweichungen und sich in einer Lage zu befinden, in der es leicht wäre, die Definition zu fixieren“ (Becker 2014:177).
} 
für die komplexen interaktionistischen Prozesse, in denen „Abweichung“ produziert wird, eröffnen möchte (Becker 2014:172).

\section{Literaturverzeichnis}

APAC-Suisse (2012). Gesetz - Recht. Internet-Seite. Zugriff am 04.09.2018 auf http://schwangerschaftsabbruch.org/infos/gesetz-recht/.

Becker, Howard (2014). Aussenseiter. Zur Soziologie abweichenden Verhaltens. In: Rössel, Jörg, Uwe Schimank und Georg Vobruba (Hg.). (2. Auflage, 25-197). Wiesbaden: Springer VS.

Boltanski, Luc (2007). Soziologie der Abtreibung. Frankfurt am Main: Suhrkamp Verlag.

Bourdieu, Pierre (1979). Entwurf einer Theorie der Praxis auf der ethnologischen Grundlage der kabylischen Gesellschaft. Frankfurt am Main: Suhrkamp Verlag.

Busch, Ulrike (2015). Vom individuellen und gesellschaftlichen Umgang mit dem Thema Abtreibung. In: Busch, Ulrike und Daphne Hahn (Hg.). Abtreibung. Diskurse und Tendenzen (13-40). Bielefeld: transcript Verlag.

Hahn, Daphne (2015). Diskurse zum Schwangerschaftsabbruch nach 1945. In: Busch, Ulrike und Daphne Hahn (Hg.). Abtreibung. Diskurse und Tendenzen (41-59). Bielefeld: transcript Verlag.

Kuttner, Heinz-Georg (2008). Zur Soziologie der Abtreibung. Dziecko. Studium interdyscyplinarne, Lublin, 503-530.

Lamnek, Siegfried (2013). Theorien abweichenden Verhaltens I. „Klassische Ansätze“. Paderborn: Wilhelm Fink.

Rössel, Jörg, Uwe Schimank und Georg Vobruba (2014). Einleitung. Devianzsoziologie, Labeling und die Nonchalance des Interaktionisten: Howard Beckers bescheidener und zentraler Beitrag zur Devianzsoziologie. In: Rössel, Jörg, Uwe Schimank und Georg Vobruba (Hg.). Aussenseiter. Zur Soziologie abweichenden Verhaltens (2. Auflage, 7-21). Wiesbaden: Springer VS.

Schmitter, Leena (2014). Politiken der Reproduktion. Die Frauenbewegung und die Liberalisierung des Schwangerschaftsabbruchs in der Schweiz (1971-2002). Inauguraldissertation der PhilosophischHistorischen Fakultät der Universität Bern, Bern.

Schweiger, Petra (2015). Schwangerschaftsabbruch. Erleben und Bewältigen aus psychologischer Sicht. In: Busch, Ulrike und Daphne Hahn (Hg.). Abtreibung. Diskurse und Tendenzen (235-256). Bielefeld: transcript Verlag.

Schweizerisches Strafgesetzbuch StGB (1937). Art 119, Stand am 1. März 2018. Internet-Seite. Zugriff am 06.09.2018 auf https://www.admin.ch/opc/de/classified-compilation/19370083/index.html. 